\title{
Functional analysis of RIP toxins from the Drosophila endosymbiont Spiroplasma poulsonii
}

\author{
Mario Gonzalo Garcia-Arraez ${ }^{1 *}$ (D) Florent Masson ${ }^{1}$ (D), Juan Camilo Paredes Escobar ${ }^{2}$ (D) and Bruno Lemaitre ${ }^{1 *}$ (D)
}

\begin{abstract}
Background: Insects frequently live in close relationship with symbiotic bacteria that carry out beneficial functions for their host, like protection against parasites and viruses. However, in some cases, the mutualistic nature of such associations is put into question because of detrimental phenotypes caused by the symbiont. One example is the association between the vertically transmitted facultative endosymbiont Spiroplasma poulsonii and its natural host Drosophila melanogaster. Whereas S. poulsonii protects its host against parasitoid wasps and nematodes by the action of toxins from the family of Ribosome Inactivating Proteins (RIPS), the presence of S. poulsonii has been reported to reduce host's life span and to kill male embryos by a toxin called Spaid. In this work, we investigate the harmful effects of Spiroplasma RIPs on Drosophila in the absence of parasite infection.

Results: We show that only two Spiroplasma RIPS (SpRIP1 and SpRIP2) among the five RIP genes encoded in the S. poulsonii genome are significantly expressed during the whole Drosophila life cycle. Heterologous expression of SpRIP1 and 2 in uninfected flies confirms their toxicity, as indicated by a reduction of Drosophila lifespan and hemocyte number. We also show that RIPs can cause the death of some embryos, including females.
\end{abstract}

Conclusion: Our results indicate that RIPs released by S. poulsonii contribute to the reduction of host lifespan and embryo mortality. This suggests that SpRIPs may impact the insect-symbiont homeostasis beyond their protective function against parasites.

Keywords: Spiroplasma, Endosymbiosis, Ribosome inactivating protein, Drosophila

\section{Background}

Endosymbiosis refers to a persistent interaction between two partners, generally a eukaryotic host and a microbial symbiont that lives within the host's body. Such interactions are particularly frequent in insects, of which more than half of species are estimated to harbor at least one endosymbiont [1, 2]. Insect endosymbionts can affect their host in multiple ways, including beneficial effects such as metabolic complementation, heat tolerance or protection against viruses and parasites [3-7]. However some endosymbiotic associations can also have detrimental consequences for the insect fitness, such as a decreased lifespan or fertility $[8,9]$. Among the most widespread facultative endosymbionts that manipulate

\footnotetext{
* Correspondence: mario.garciaarraez@epfl.ch; bruno.lemaitre@epfl.ch ${ }^{1}$ Global Health Institute, School of Life Science, École Polytechnique Fédérale de Lausanne (EPFL), Lausanne, Switzerland

Full list of author information is available at the end of the article
}

insect reproduction are the genera Wolbachia and Spiroplasma [10].

Spiroplasma poulsonii (hereafter Spiroplasma) is a natural symbiont of the fruit fly Drosophila melanogaster [11-13]. It lives extracellularly in the fly hemolymph and is vertically transmitted by trans-ovarial transfer. Spiroplasma colonizes the germline during vitellogenesis by co-opting the yolk transport and internalization machinery [14]. Intriguingly, it completely lacks a cell-wall and thus immunogenic surface molecules, such as peptidoglycan, which renders it invisible for the host immune system [15-19]. In adult flies, Spiroplasma grows over time reaching a titer of $10^{5}-10^{6}$ bacteria per $\mu$ of hemolymph [20]. Spiroplasma infection shortens the lifespan of Drosophila, suggesting that either the bacteria causes damages only at high titer, or that the damages take time to kill the host [19]. Interestingly, the growth of S. poulsonii is limited by the availability of

(c) The Author(s). 2019 Open Access This article is distributed under the terms of the Creative Commons Attribution 4.0 International License (http://creativecommons.org/licenses/by/4.0/), which permits unrestricted use, distribution, and reproduction in any medium, provided you give appropriate credit to the original author(s) and the source, provide a link to the Creative Commons license, and indicate if changes were made. The Creative Commons Public Domain Dedication waiver (http://creativecommons.org/publicdomain/zero/1.0/) applies to the data made available in this article, unless otherwise stated. 
host lipids, preventing its overgrowth in condition of nutrient scarcity [19].

One of the most striking phenotypes caused by Spiroplasma is male-killing, whereby infected male embryos die during their development while most infected females survive [12]. As Spiroplasma is only transmitted by female flies, male-killing is thought to favor the spread of the bacteria among host natural populations [21]. Recently, a Spiroplasma toxin containing Ankyrin-repeats, named Spiroplasma Androcidin (Spaid), has been described as a crucial male-killing agent [22, 23]. Heterologous expression of Spaid in uninfected flies is sufficient to kill males. Moreover, its expression during early embryogenesis induces DNA-damage-dependent apoptosis and defective neurogenesis in uninfected male embryos, which fully recapitulates male-killing phenotypes [24-27].

Studies have shown that in some contexts, Spiroplasma can also provide a benefit to its host as they mediate protection against parasitoid wasps and nematodes in several Drosophila species [28-31]. Protection is a major ecological benefit that can lead to a fast spreading of Spiroplasma in wild populations [32]. Two complementary mechanisms have been implicated in Spiroplasma protection against parasites: a metabolic competition for host lipids between Spiroplasma and the parasites, and Spiroplasma production of Ribosome-Inactivating Proteins (RIPs) that damage ribosomes of both wasp eggs and nematodes [28, 30,31]. RIPs are found in plants and bacteria, where they act as a defense against eukaryotic parasites [30, 31, 33-35]. They recognize a conserved region of the $28 \mathrm{~S}$ ribosomal RNA called the Sarcin-Ricin Loop (SRL). The secondary structure of the SRL consists in a hairpin loop displaying an adenine that is necessary for protein synthesis [36]. RIPs cleave the central adenine from the SRL in a process called depurination, thus blocking protein synthesis [37].

In this article we investigated the role of RIPs produced by the facultative endosymbiont $S$. poulsonii (hereafter $S p R I P s)$ in its natural host $D$. melanogaster. Similarly to the reduced lifespan observed in infected flies, we show that heterologous expression of SpRIPs coding genes in uninfected flies shortens their life span. Furthermore, uninfected-embryos expressing SpRIPs have high mortality rate and a female-biased sex-ratio among the surviving individuals, suggesting that males may be more sensitive to the ectopic expression of this toxin.

\section{Results}

\section{SpRIPs depurinate the 28S rRNA of $D$. melanogaster}

$S$. poulsonii genome contains five genes encoding RIPs (SpRIP1-5) [20, 30, 31, 38]. All of them have a signal peptide, suggesting a secretion of the mature protein, and a conserved N-glycosidase domain in charge of the depurination reaction [30, 31]. All copies are chromosomal, suggesting that they are very stable compared to other endosymbiont toxin coding genes that are located on plasmids or mobile elements such as Spaid in S. poulsonii or the cytoplasmic incompatibility factor of Wolbachia [20, 23, 39]. Transcriptome analysis has shown that only two of them, SpRIP1 and SpRIP2, are significantly expressed in vivo and in vitro, pointing to a possible pseudogenization of SpRIP3, 4 and 5 [20, 30].

To confirm the expression pattern of SpRIPs in infected flies, we performed RT-qPCR analysis on each of the SpRIPs. We confirmed that SpRIP1 and SpRIP2 are strongly expressed by $S$. poulsonii in D. melanogaster with no significant changes in expression level along the fly life cycle (Fig. 1a and b). Very low levels of SpRIP 3, 4 and 5 transcripts were detected, in accordance with the literature (Additional file 1: Figure S1). We then measured RIP activity using a RT-qPCR assay. This assay relies on the ability of reverse transcriptases to incorporate a thymine in complementary DNA in place of the void position present on the depurinated RNA molecule. It is then possible to design primers that bind specifically to the intact cDNA (containing an adenine) or to the depurinated one (containing a thymine) [31]. Comparisons between infected and uninfected flies confirmed that $S$. poulsonii depurinates the 28S rRNA of Drosophila, as previously shown for larvae and 1 week old adult flies [30]. Monitoring RIP activity along the whole Drosophila lifecycle revealed particularly high levels of depurination in embryos and old adult flies (Fig. 1c). A control assay using primers amplifying fragments outside of the SRL showed that the total number of 28 rRNA transcripts was the same between infected and uninfected flies (Fig. 1d). As the level of expression of SpRIP in Spiroplasma is constant, the high level of depurination in embryos and old adult flies likely results from the higher Spiroplasma titer in the host at these stages [19].

\section{SpRIP1 and SpRIP2 expression is toxic for Drosophila melanogaster}

We generated four different transgenic fly lines expressing singly $S p R I P 1, S p R I P 2, \mathrm{BiP}+\operatorname{SpRIP1}$ or $\mathrm{BiP}+\operatorname{SpRIP2}$ under the control of the GAL4/UAS system [40]. BiP is a signal peptide used to trigger the secretion of proteins in D. melanogaster $[41,42]$. The toxicity of these constructs was tested using the "Rough Eye Phenotypes" (REP) assay, which allows to study the activity of a putative toxin driven by an eye-specific driver (ey-GAL4) to observe eventual deleterious effect of the protein on this organ's structure $[43,44]$. The REP assay allows to study toxin activity by monitoring defects including loss of bristles, fusion of ommatidias, necrosis, loss of pigmentation and reduced eye size [43, 45]. All control flies developed a normal eye structure. On the contrary, flies expressing UAS-SpRIPs under ey-GAL4 control developed 
A

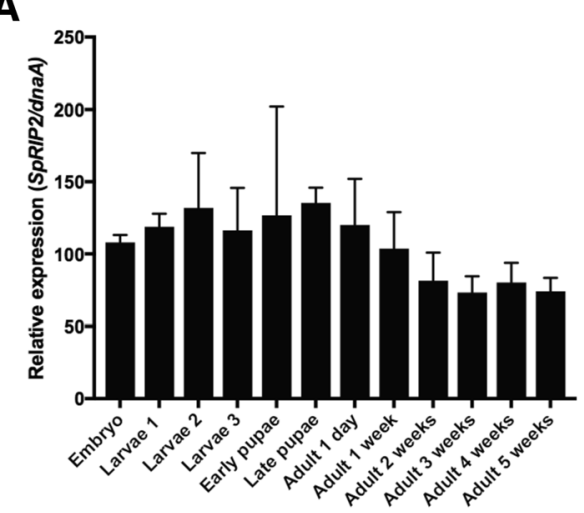

C

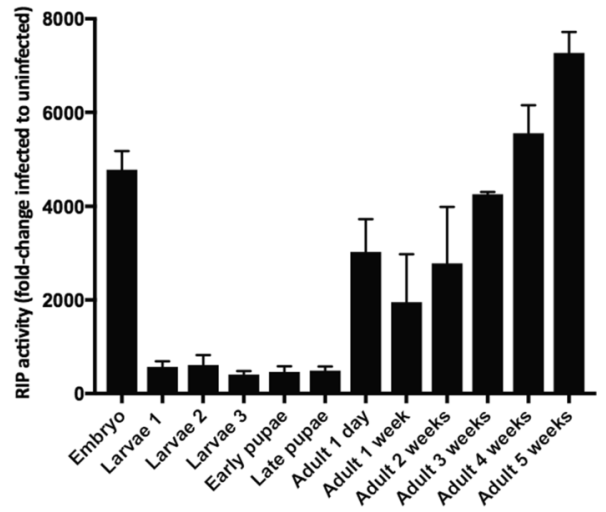

B

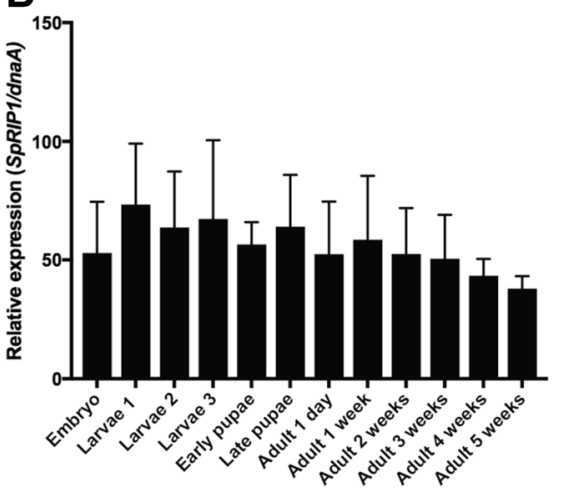

D

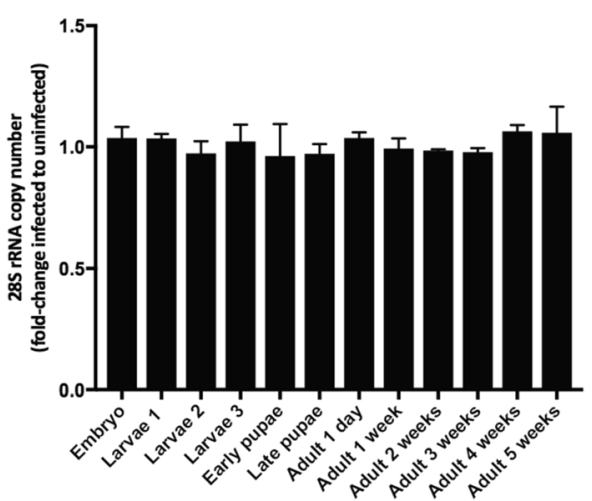

Fig. 1 a SpRIP1 expression level in infected flies along Drosophila development stages (One way ANOVA; development stage $p=0.9055)$. b SpRIP2 expression level in infected flies along Drosophila development stages (One way ANOVA; development stage $p=0.5129$ ). c RIP activity in infected flies compared to uninfected flies (Two way ANOVA; Spiroplasma infection $p^{* * *}<0.0001$; development stages $p^{* * *}<0.0001$; interaction $p^{* * *}<0.0001$ ). d Intact 285 rRNA quantification in infected versus uninfected flies along Drosophila development stages

a reduced eye along with severe abnormalities, and in some cases no eye at all (Fig. 2). This demonstrates that both $S p$ RIP1 and 2 act as toxins on Drosophila cells.

\section{Ectopic expression of SpRIP1 and SpRIP2 decreases uninfected flies life span}

Spiroplasma-infected flies have a shorter lifespan compared to uninfected ones [16]. Moreover, old infected flies have been reported to have a decreased climbing activity which suggests neurological damages [19]. We first confirm this phenotype, observing that infected flies have a lifespan reduced by about 20 days (Fig. 3 and Additional file 2: Figure S2). As Spiroplasma resides in the hemolymph, we hypothesized that the impact of Spiroplasma on host lifespan could be due to accumulation of a toxin released in the hemolymph. Accordingly, proteomics analysis of hemolymph of 2 weeks old Spiroplasma-infected flies revealed the presence of SpRIP1 and $S p$ RIP2 (S. Rommelaere, F. Masson, and B. Lemaitre, unpublished data).

To further address the impact of SpRIPs on Drosophila viability, we tested the effect of ectopic expression of
SpRIPs on the life span of uninfected flies. Drosophila expressing $S p R I P 1$ or $B i P+S p R I P 1$ constructs did not develop further than larval instars, preventing the use of these constructs for lifespan analysis. Interestingly, uninfected flies expressing SpRIP2 and BiP + SpRIP2 had a markedly decreased lifespan by about 30 days in average compared to uninfected flies, which live about 75 days (Fig. 3; Logrank test $p^{* * * *}<0.0001$ ). The lifespan of these transgenic lines was also shorter than the one of infected flies, which live about 45 days (Fig. 3; Logrank test $p^{\text {***** }}$ $<0.0001)$. The lifespans seem to be depending on the expression level and activity of the RIPs (Additional file 3: Figure S3 and Additional file 4: Figure S4).

To further test the implication of $S p$ RIPs in premature adult lethality, we generated a transgenic fly line expressing a $1492 \mathrm{bp}$ fragment of the $28 \mathrm{~S}$ rRNA under the control of a UAS upstream sequence [40]. This fragment contains the conserved SRL targeted by RIPs and was designed to buffer RIP activity by increasing the number of targets for the toxin, thus working as an antidote. Spiroplasma-infected flies with ubiquitous expression of SRL fragment display an increase in their lifespan by 

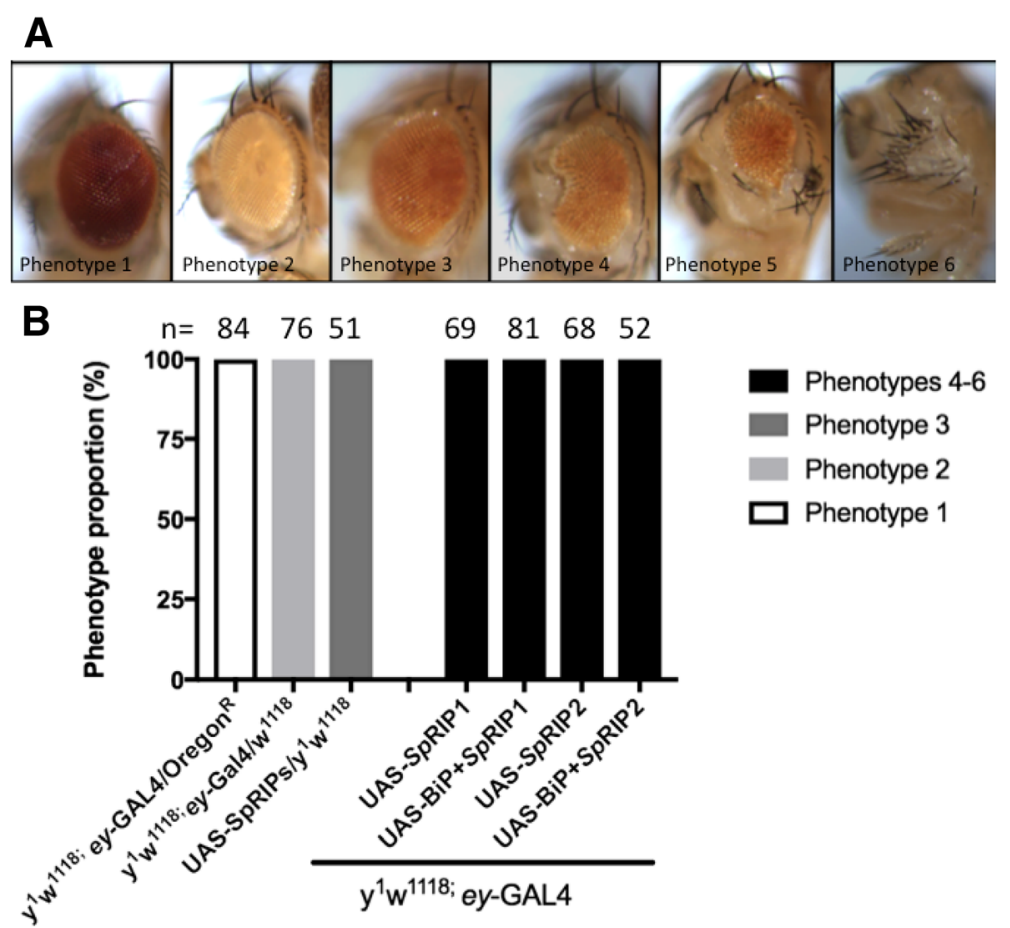

Fig. 2 Rough Eye Phenotype assay. a Representative bright-field images of Drosophila eye phenotypes obtained during the assay. $\mathbf{b}$ Percentage of each phenotype. $n$ indicates the number of flies obtained for each cross. Each cross has been repeated three independent times

about 5 days compared to infected wild-type flies (Fig. 3; Logrank test $\left.p^{* * * * *}<0.0001\right)$. Collectively, these results are consistent with the implication of SpRIP in shortening Drosophila life span.

Spiroplasma-infected flies and uninfected flies expressing SpRIP2 have reduced hemocyte count

As $S$. poulsonii is found in the fly hemolymph, we hypothesized that hemocytes should be the most affected cell type by RIP toxins. We thus visualized hemocyte in Spiroplasma-infected and uninfected adult flies, carrying the hemocyte marker Hml-GAL4 > UAS-GFP. In uninfected adult flies, sessile hemocytes are found in patches beneath the cuticle in the middle of the dorsal abdomen [46]. Interestingly, Spiroplasma-infected flies have reduced number of sessile patches (Fig. 4a). To confirm this observation, we indirectly estimated the number of hemocytes in adult flies by monitoring the expression of

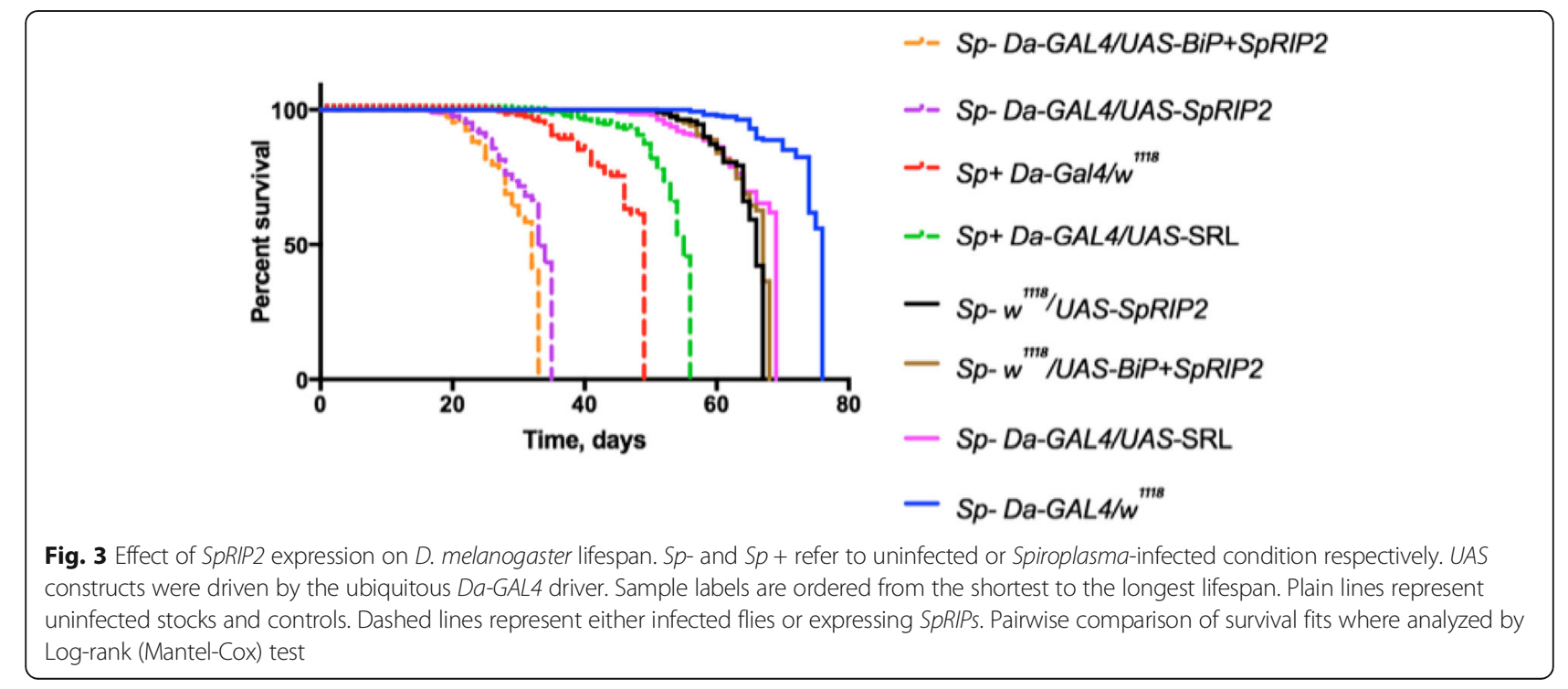


hemolectin $(\mathrm{hml})$, a gene which expression is hemocyte-specific. Consistent with a reduction of the number of hemocytes, the expression of $h \mathrm{ml}$ was halved in Spiroplasma-infected flies compared to uninfected ones in two different wild type strains (Fig. 4b). We conclude that the presence of Spiroplasma greatly reduces the number of hemocytes. This reduction could reflect the shortening of lifespan as a decreased hemocyte count is one of the hallmark of aging in flies [47]. To test whether $S p$ RIPs could mediate this effect, we monitored the level of hemocytes in adult flies expressing $S p R I P 2$ and BiP + SpRIP2 under the control of two ubiquitous GAL4 drivers. $\mathrm{Hml}$ expression quantification revealed a decrease in the number of hemocytes in these flies similar to the decrease observed upon Spiroplasma infection (Fig. 4c and Additional file 5: Figure S5). These results suggest that $S p R I P s$ cause hemocytes death, which in turn could contribute to aging and premature death of flies.

\section{SpRIPs ectopic expression causes embryo mortality} revealing higher in male embryos compare to female ones

We have shown that RIP activity is particularly high in Spiroplasma-infected embryo compared to other developmental stages (Fig. 1c) raising the possibility that $S p R I P 1$ and $S p R I P 2$ could contribute to embryo mortality. To test this possibility, we first monitored the effect of the ectopic expression of SpRIPs in uninfected individuals by using either the ubiquitous zygotic Da-GAL4 driver in embryos or the maternal driver MTD-GAL4. We monitored embryo mortality as the percent of embryos that do not hatch, which is about 5\% in uninfected wild type embryos (Fig. 5a). All uninfected embryos with ectopic expression of SpRIP1 or BiP + SpRIP1 die, reflecting the high toxicity of SpRIP1. However, the expression of UASpSpRIP2 kills about $70 \%$ of the embryos (Dunnett's multiple comparison test against uninfected w1118 $\left.p^{* *}<0.0074\right)$. Interestingly, over-expression of UASp$B i P+S p R I P 2$ shows a lower toxicity with a mortality rate up to 30\% (Dunnett's multiple comparison test against uninfected $w 1118 p^{* * * *}<0.0001$ ) (Fig. 5a). We hypothesized that the secretion of the toxin out of the embryo's cells reduces its toxicity. To reinforce the hypothesis that RIP activity is indeed responsible for embryo death, we measured RIP activity during embryogenesis for each construct. We observed a correlation between the level of RIP activity and the mortality (Pearson's correlation test $p^{* * * *}<0.001$ )
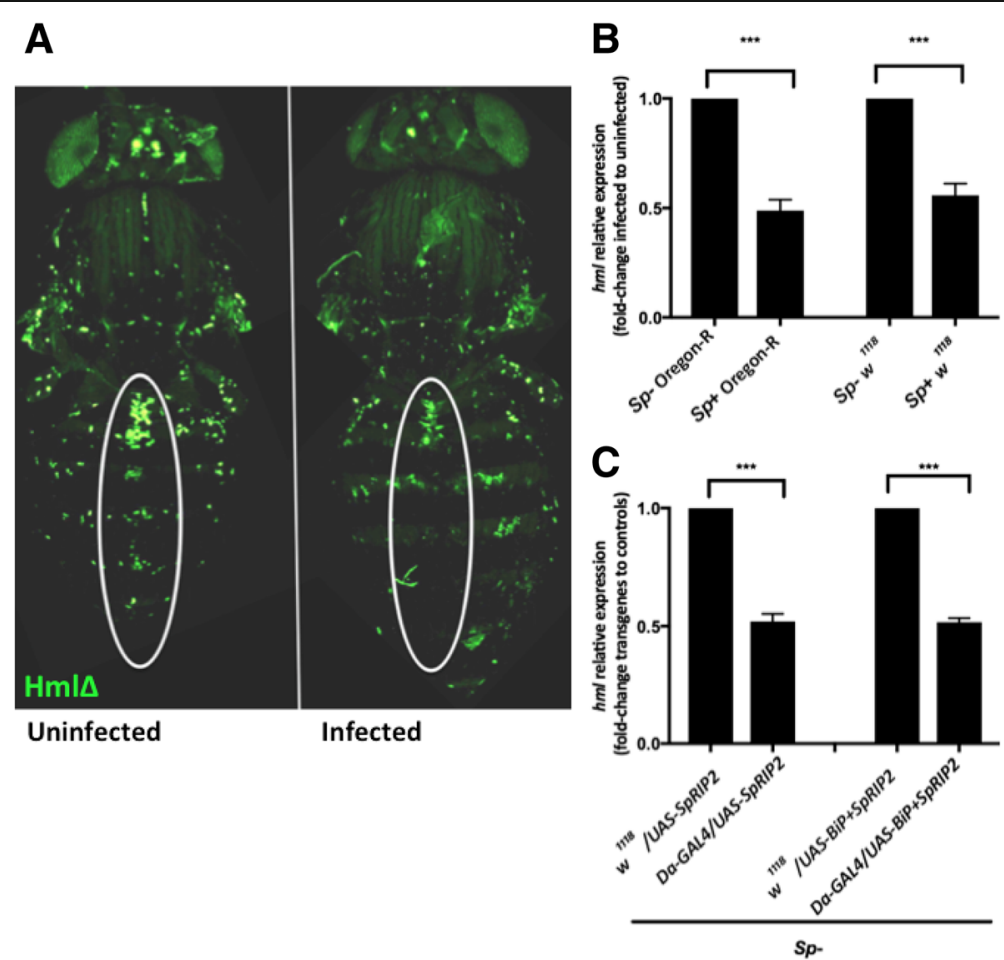

Fig. 4 a Confocal image of HmIS-GAL4/UAS-GFP adult flies. In uninfected flies, hemocyte patches are mostly located within the white circle, following the antero-posterior axis. In infected flies only a few patches remain and have lower fluorescence intensity. $\mathbf{b} \mathrm{hm} /$ transcription level in infected and uninfected adult wild type flies (Dunnett's multiple comparisons test $p^{* * *}<0.0001$ ). $\mathbf{c ~ h m / ~ t r a n s c r i p t i o n ~ l e v e l ~ i n ~ l i n e s ~ e x p r e s s i n g ~ U A S - S p R I P 2 ~ a n d ~ U A S - ~}$ $B i P+S p R I P 2$ under Da-GAL4 control. Expression of both constructs lead to a decrease in hemocyte number (Dunnett's multiple comparisons test $p^{* * *}$ $<0.0001)$. Sp- and Sp + refer to uninfected or Spiroplasma-infected condition respectively. Controls are normalized as 1 


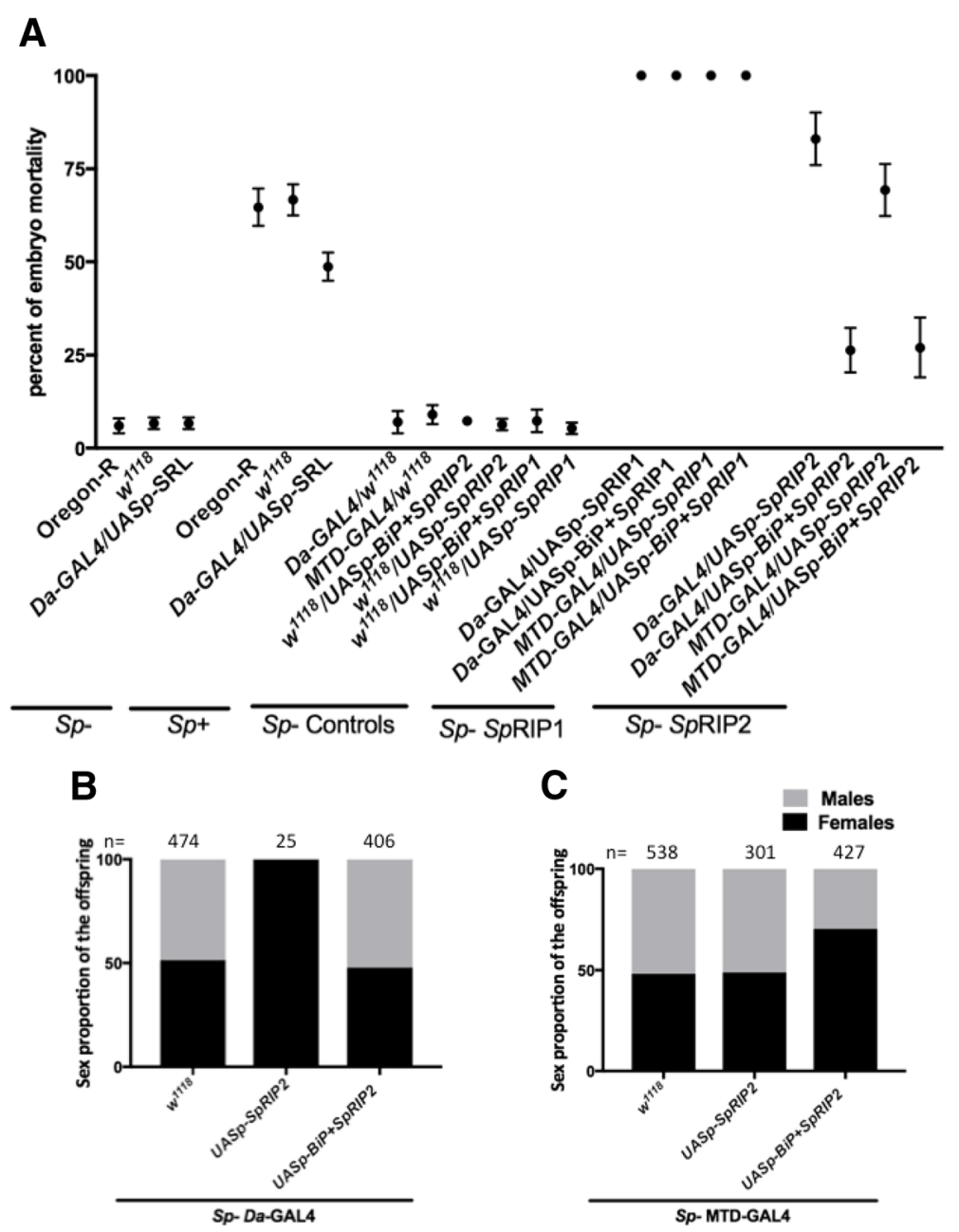

Fig. 5 a Effect of SpRIP expression on embryo mortality. b Sex ratio of uninfected flies expressing UASp-SpRIP2 under Da-GAL4 control (ubiquitous). $\mathbf{c}$ Sex ratio of uninfected flies expressing UASp-SpRIP2 under MTD-GAL4 control (maternal specific). $n$ indicates the number of adult flies counted for the assay. $S p$ - and $S p+$ refer to uninfected or Spiroplasma-infected condition respectively

(Additional file 6: Figure S6), suggesting that the mortality indeed results from RIP activity.

To further test the possible implication of $S p$ RIPs in embryo mortality, we took advantage of the UASp-SRL construct by analyzing whether buffering RIP activity with additional SRL target could rescue Spiroplasma-infected embryos from dying. We first observed that embryonic lethality reaches about $65 \%$ in Spiroplasma-infected flies, well above the expected $50 \%$ if males only were dying. This suggests that not only does Spiroplasma kill males, but also a small fraction of the female progeny, roughly estimated at $12.5 \%$ (Dunnett's multiple comparison test $p^{* * * *}$ $<0.0001$ ). Interestingly, ectopic expression of SRL slightly decreases mortality of infected embryos by $10 \%$ (Dunnett's multiple comparison test $p^{* * *}<0.0001$ ) (Fig. 5a). The sex ratio of the surviving embryo was still $100 \%$ females, suggesting that the buffering of RIP activity by the UASp-SRL construct is sufficient to rescue females but not males (Fig. 5a).

While the sex-ratio of uninfected hatching flies is of 50\% females and 50\% males (Fig. 5b, c and Additional file 7: Figure S7), the sex-ratio of uninfected D. melanogaster hatching flies from embryos expressing SpRIP2 were biased toward female. It ranged from $70 \%$ when the construct is under the control of the maternal driver MTD (Fisher's exact test $\left.p^{* * * *}<0.001\right)$ to $100 \%$ when the ubiquitous driver Da-GAL4 was used (Fisher's exact test $p^{* * * *}<0.001$ ) (Fig. 5b and c), suggesting that males are more sensitive to RIP activity.

\section{Discussion}

S. poulsonii protects its host against macro-parasites such as nematodes and parasitoid wasps and RIP toxins has been shown to play a major role in this protection 
[30, 31]. In this study, we provide evidence that Spiroplasma RIPs could affect symbiosis beyond their implication in endosymbiont-mediated protection by harming the host and contributing to lifespan shortening and embryo mortality.

We show that only two out of the five putative RIP genes contained in Spiroplasma genome are expressed all along the life cycle of Drosophila with peaks during embryogenesis and late adulthood. As S. poulsonii titer increases with time [19], we hypothesize that the peak in old adults is a consequence of the high density of Spiroplasma in the host hemolymph, rather than a change in the gene expression in the bacteria. Infected embryos also reveal particularly high RIP activity, likely due the transmission of RIPs and of already depurinated ribosomes from the mother. Our over-expression studies were carried out with the ubiquitous Da-GAL4 driver as it revealed the closest expression level to natural infection. Such studies confirm that SpRIP1 and SpRIP2 target the 28S rRNA of its host as previously shown [30]. Transgenic fly lines expressing SpRIP1 display a higher mortality rate for embryos and larva than those expressing $S p R I P 2$, which may result from a higher RIP1 transgene expression (Additional file 3: Figure S3). Last, the addition of a Drosophila secretion signal to the protein sequence tends to reduce its toxicity, which is consistent with $S p$ RIPs targeting $28 S$ rRNA within the cells.

Previous studies have shown that Spiroplasma shortens the fly lifespan but the underlying mechanism was unknown, although the synthesis of cardiolipins by the bacteria has been proposed as a cause [19]. Our results suggest that Spiroplasma RIPs contribute to the premature death of infected flies. According to this model, the increasing Spiroplasma titer in aging flies is accompanied by an increase of $S p$ RIP release in the fly hemolymph, which eventually damages the host tissues. Ectopic expression of SpRIPs within cells can be more toxic than natural bacterial expression, as ribosomes are then more exposed to the toxin. This situation is however happening naturally only during the first $3 \mathrm{~h}$ of embryogenesis (before the cellularization), after which Spiroplasma is extracellular. The addition of a Drosophila secretion signal to the toxin thus better mimics the natural situation. However, Drosophila life span remained shortened even upon SpRIP secretion, suggesting that the toxin is able to enter the cells and depurinate ribosomes efficiently.

Similarly to the phenotype of Spiroplasma-infected flies, we show that over-expression of SpRIP1 and SpRIP2 are associated with an increase in embryo lethality, a shorter lifespan and a decrease in hemocytes number. While $S p$ RIPs contribute to the protection against Drosophila's parasites, our study suggests that these toxins have also a strong detrimental effect in the host with a tangible impact in late adulthood. This suggests that Spiroplasma has not developed any mechanism to shut down RIP expression at the adult stage or in absence or parasite infections. Maintaining a constitutive $S p$ RIP production could be a way to react as quickly as possible to parasite infections at a low cost for the host. It is indeed likely that the fitness cost associated with lifespan reduction in Drosophila is minimal, as most eggs are laid during the first 2 weeks [48].

\section{Conclusion}

Insect endosymbioses encompass a continuum of interactions ranging from mutualism to parasitism. In some cases however, assessing the beneficial or detrimental nature of the interaction for the host can be delicate. The Spiroplasma/Drosophila symbiosis is a prime example of such versatile ecological outcome: the bacteria protects its host against widespread parasites, conferring a major ecological benefit, but also kills male progeny and drastically reduces the adults lifespan, reflecting a pathogenic interaction. SpRIPs are involved in these two different faces of Spiroplasma endosymbiosis. They are directly involved in host protection against parasites, but can also cause strong damage to the host in absence of parasite infection, making them the first described endosymbiont-encoded toxins to directly harm its adult host.

\section{Methods}

\section{Fly stocks and handling}

Infected lines were generated in 2011 by injection of Spiroplasma-infected hemolymph in Oregon-R females. [17]. Infected lines have been maintained in the laboratory establishing genetically identical lines of Oregon- $R$ Spiroplasma infected and uninfected. Hemocytes were observed on 4 weeks old females w1118; Hml $\triangle G A L-4$ > $U A S-G F P$ [49]. For all the experiments, flies were maintained at $25^{\circ} \mathrm{C}$ on standard cornmeal medium. Embryos were collected from 5 to 7 days old flies by using cages and yeasted grape juice plates. Lifespan experiments were done as described in [17]. The driver for REP assay (ey-GAL4) was obtained from Bloomington stock center (\#8221). All experiments have been repeated three independent times.

\section{RNA, DNA extractions and RT-qPCR}

RNA, DNA extractions, and RT-qPCR were performed as described in $[17,19,28]$. Reverse transcription was done using $500 \mathrm{ng}$ of RNA per sample, which was isolated from 3 adult flies, 3 larvae, 3 pupae, or 100 to 300 embryos. SpRIPs expression and activity were measured along the whole life cycle by RT-qPCR. Expression for each $S p R I P$ was analyzed individually except for $S p R I P 3$, SpRIP4 \& SpRIP5 that were measured with a single pair of primers because of their high sequence identity. RT-qPCR calculations for the expression level of SpRIPs 
was done following the $\triangle \mathrm{CT}$ method normalizing by $d n a A$ expression level. Primers for $d n a A$ are described in [17]. RT-qPCR calculations to compare the expression level of SpRIPs between the transgenic fly lines and Spiroplasma-infected flies was done following the $\triangle \mathrm{CT}$ method normalizing by rps17 expression level. Primers for UASSpRIP1 expression are Forward: 5' - CGT AGC AGGT GGTGTTGTTC-3' Reverse: 5'- GCTTCACCCACATC AGCAAG-3' (efficiency = 1.81). Primers for UAS-SpRIP2 expression are Forward: 5' - CGT AGC TCGATACCA GCGTGACCATC-3' Reverse: 5' - CGTTCTGCAGGTTG TACTCG-3' (efficiency = 1.94). RIP activity assay was performed as described in [31]. All calculations for RIP activity and hemocyte count have been done following the $\Delta \Delta C T$ method and these figures represent the fold change between the experimental condition samples and the controls which are valued as 1 [50]. Primers for $h m l$ are: Forward: 5'-GAGCACTGCATACCCCTACC-3' Reverse: 5'-CCGTGCTGGTTACACTCCTT-3' (efficiency = 1.88). Gene expression levels were normalized to rps17. Figures and statistical results were obtained using GraphPad Prism 7.0b software. All experiments have been repeated three independent times.

\section{Design and construction of UAS-SPRIP1 and UAS-SpRIP2 constructs}

Spiroplasma has an alternative genetic code and a strong codon bias compared to Drosophila [38]. SpRIP1 and SpRIP2 gene sequences were codon optimized for insect translation using Geneious v8.1.9. The secretion signal from the sequence of $\mathrm{BiP}$ (Hsc70-3) was added at the 5' end of the RIP genes flanked by two BglII restriction sites. The optimized BiP-SpRIP1 and 2 were fully synthesized and cloned in a pDONR221 vector for Gateway cloning by Invitrogen GeneArt gene synthesis services. Optimized SpRIP1 and 2 were obtained from BiP+ SpRIP 1 and 2 by digestion of the BiP sequence by BglII and re-ligation of the plasmid on itself. The fragment of $28 \mathrm{~S}$ rRNA was amplified from Oregon-R flies and also cloned in pDONR221. All transgenes were cloned into a $U A S p$ and a UASt vector by Gateway LR reaction and injected in $D$. melanogaster w1118 embryos by Bestgene Inc., Chino Hills, USA.

\section{Embryo mortality assay}

A total of 100 embryos were collected per genotype on grape juice plates 15-20 h after egg laying. After ten more hours, the remaining embryos that did not hatch (dead) were counted. Experiments were done simultaneously with two different drivers, the ubiquitous $D a-G A L 4$, and the maternal MTD-GAL4. All experiments have been repeated three independent times.

\section{Additional files}

\begin{abstract}
Additional file 1: Figure S1. SpRIPs 3,4 and 5 expression level in infected flies along Drosophila development stages (One way ANOVA; development stage $p=0.9992$ ). (TIF $1384 \mathrm{~kb}$ )
\end{abstract}

Additional file 2: Figure S2. Lifespan of infected and uninfected wild types flies (controls for Fig. 3). Sp- and Sp + refer to uninfected or Spiroplasma-infected condition respectively. (TIF $1983 \mathrm{~kb}$ )

Additional file 3: Figure S3. Expression level of (A) SPRIP1 and (B) $S P R I P 2$ in embryos from uninfected, infected and transgenic fly lines expressing UAS-RIP normalized by host rsp 17 transcript level (One way ANOVA; $p^{* *}=0.0031$ for SpRIP1 and $p^{* *}=0.0049$ for SpRIP2). (C) Expression level of SpRIP2 in adults from uninfected, infected and transgenic fly lines expressing UAS-RIP normalized by host rsp 17 transcript level (One way ANOVA; $\left.p^{* *}<0.0081\right)$. (TIF $5534 \mathrm{~kb}$ )

Additional file 4: Figure S4. (A) Comparison of RIP activity in Spiroplasma-infected embryos with uninfected transgenic embryos expressing SpRIPS (One way ANOVA; SpRIP1 $p^{* * *}=0.001$; One way ANOVA; $\left.S p R I P 2 p^{* *}=0.0021\right)$. (B) Intact $28 \mathrm{~S}$ rRNA quantification in infected embryos and uninfected transgenic fly lines (C) Comparison of RIP activity in Spiroplasma-infected adult flies with uninfected transgenic adult fly (One way ANOVA; SpRIP2 $p^{* * *}=0.001$ ) (D) Intact 285 rRNA quantification in infected adult flies and uninfected transgenic adult flies. (TIF $6378 \mathrm{~kb}$ )

Additional file 5: Figure S5. $\mathrm{hm} /$ transcription level in lines expressing UAS-SpRIP2 and BiP + SPRIP2 under actin-GAL4 control. Expression of both constructs also leads to a decrease in hemocyte number (Dunnett's multiple comparisons test $\left.p^{* * *}<0.0001\right)$. Controls are normalized as 1 . (TIF $1147 \mathrm{~kb}$ )

Additional file 6: Figure S6. Correlation between RIP activity in embryos aged 0 to $24 \mathrm{~h}$ after egg laying and embryo mortality (Pearson's test $p<0.0001)$. RIP activity in infected wild types was normalized by uninfected samples. Transgenic fly lines were normalized by Da-GAL4/ w1118. Controls are normalized as 1. (TIF $3499 \mathrm{~kb}$ )

Additional file 7: Figure S7. Sex ratio of the control fly lines for Fig. 5. $S p$ - and $S p+$ refer to uninfected or Spiroplasma-infected condition respectively. (TIF $4019 \mathrm{~kb}$ )

\section{Abbreviations}

ARP: Adhesion Related Proteins; qPCR: Quantitative Polymerase Chain Reaction; REP: Rough Eye Phenotypes; RIP: Ribosome Inactivating Protein; RT: Reverse Transcription; Spaid: S. poulsonii androcidin; SpRIP: S. poulsonii Ribosome Inactivating Protein

\section{Acknowledgments}

We sincerely thank Steve J. Perlman (Department of Biology, University of Victoria, Canada) along with two people from his laboratory, Matthew J. Ballinger and Phineas T. Hamilton for the constructive discussions and the sharing of information. We also thank Maroun Bou Sleiman, Samuel Rommelaere and Toshiyuki Harumoto (School of Life Science, École Polytechnique Fédérale de Lausanne) for their comments and discussion about this project.

Funding

This work was funded by ERC (European Research Council) advanced grant no. 339970 and the SNF (Swiss National Science Foundation) Sinergia grant no. CRSII3_154396.

Availability of data and materials

All data generated or analyzed during this study are included in this published article (and its additional files).

\section{Authors' contributions}

GGA performed fly handling assays and RT-qPCR experiments and drafted the manuscript. FM collaborated in the creation of transgenic fly lines. FM, JCP and BL supervised the project and contributed to the manuscript. All authors read and approved the final manuscript. 


\section{Ethics approval and consent to participate}

Not applicable.

\section{Consent for publication}

Not applicable.

\section{Competing interests}

The authors declare that they have no competing interests.

\section{Publisher's Note}

Springer Nature remains neutral with regard to jurisdictional claims in published maps and institutional affiliations.

\section{Author details}

1 Global Health Institute, School of Life Science, École Polytechnique Fédérale de Lausanne (EPFL), Lausanne, Switzerland. ${ }^{2}$ International Centre of Insect Physiology and Ecology (ICIPE), Kasarani, Nairobi, Kenya.

Received: 9 July 2018 Accepted: 31 January 2019

Published online: 20 February 2019

\section{References}

1. Kikuchi Y. Endosymbiotic Bacteria in insects: their diversity and Culturability. Microbes Environ. 2009;24:195-204.

2. Ishikawa H. Insect Symbiosis. An introduction; 2003.

3. Ferrari J, Vavre F. Bacterial symbionts in insects or the story of communities affecting communities. Philos Trans R Soc B. 2011;366:1389-400,

4. Teixeira L, Ferreira Á, Ashburner M. The bacterial symbiont Wolbachia induces resistance to RNA viral infections in Drosophila melanogaster. PLoS Biol. 2008;6:e1000002.

5. Vigneron A, Masson F, Vallier A, Balmand S, Rey M, Vincent-Monégat C, et al. Insects recycle endosymbionts when the benefit is over. Curr Biol. 2018;24: 2267-73.

6. Montllor CB, Maxmen A, Purcell AH. Facultative bacterial endosymbionts benefit pea aphids Acyrthosiphon pisum under heat stress. Ecol Entomol. 2002:27:189-95.

7. Dunbar HE, Wilson ACC, Ferguson NR, Moran NA. Aphid thermal tolerance is governed by a point mutation in bacterial symbionts. PLoS Biol. 2007;5:e96

8. Ciche TA, Darby C, Ehlers R-U, Forst S, Goodrich-Blair H. Dangerous liaisons: the symbiosis of entomopathogenic nematodes and bacteria. Biol Control. 2006;38:22-46.

9. Loreto RG, Hughes DP. Chapter eight - disease dynamics in ants: a critical review of the ecological relevance of using generalist Fungi to study infections in insect societies. In: Genetics and molecular biology of Entomopathogenic Fungi; 2016. p. 287-306.

10. Hamilton PT, Perlman SJ. Host defense via Symbiosis in Drosophila. PLoS Pathog. 2013;9:e1003808.

11. Mateos M, Castrezana SJ, Nankivell BJ, Estes AM, Markow TA, Moran NA. Heritable endosymbionts of Drosophila. Genetics. 2006;174:363-76.

12. Counce SJ, Poulson DF. Developmental effects of the sex-ratio agent in embryos of Drosophila willistoni. J Exp Zool. 1962;151:17-31.

13. Anbutsu H, Fukatsu T. Spiroplasma as a model insect endosymbiont. Environ Microbiol Rep. 2011:3:144-53.

14. Herren JK, Paredes JC, Schüpfer F, Lemaitre B. Vertical transmission of a Drosophila endosymbiont via cooption of the yolk transport and internalization machinery. MBio. 2013;4:e00532.

15. Lemaitre B, Nicolas E, Michaut L, Reichhart J-M, Hoffmann JA. The Dorsoventral regulatory gene cassette spätzle/toll/cactus controls the potent antifungal response in Drosophila adults. Cell. 1996;86:973-83.

16. Lemaitre B, Hoffmann J. The host defense of Drosophila melanogaster. Annu Rev Immunol. 2007;25:697-743.

17. Herren JK, Lemaitre B. Spiroplasma and host immunity: activation of humoral immune responses increases endosymbiont load and susceptibility to certain gram-negative bacterial pathogens in Drosophila melanogaster. Cell Microbiol. 2011;13:1385-96

18. Hurst GDD, Anbutsu H, Kutsukake M, Fukatsu T. Hidden from the host: Spiroplasma bacteria infecting Drosophila do not cause an immune response, but are suppressed by ectopic immune activation. Insect Mol Biol. 2003;12:93-7.
19. Herren JK, Paredes JC, Schüpfer F, Arafah K, Bulet P, Lemaitre B. Insect endosymbiont proliferation is limited by lipid availability. elife. 2014;3:e02964.

20. Masson F, Copete Sandra C, Schüpfer F, Garcia-Arraez G, Lemaitre B. In vitro culture of the insect endosymbiont Spiroplasma poulsonii highlights bacterial genes involved in HostSymbiont interaction. MBio. 2018;9:1-11.

21. Hurst GDD, Crystal L. Reproductive parasitism: maternally Inherited symbionts in a Biparental world. Cold Spring Harb Perspect Biol. 2015;7: $1-20$.

22. Oishi K. Spirochaete-mediated abnormal sex-ratio (SR) condition in Drosophila: a second virus associated with spirochaetes and its use in the study of the SR condition. Genet Res. 1971;18:45-56.

23. Harumoto T, Lemaitre B. Male-killing toxin in a bacterial symbiont of Drosophila. Nature. 2018:557:252-5.

24. Martin J, Chong T, Ferree PM. Male killing Spiroplasma preferentially disrupts neural development in the Drosophila melanogaster embryo. PLoS One. 2013;8:1-8.

25. Harumoto T, Anbutsu H, Lemaitre B, Fukatsu T. Male-killing symbiont damages host's dosage-compensated sex chromosome to induce embryonic apoptosis. Nat Commun. 2016:7:12781.

26. Cheng B, Kuppanda N, Aldrich JC, Akbari OS, Ferree PM. Male-killing Spiroplasma alters behavior of the dosage compensation complex during Drosophila melanogaster embryogenesis. Curr Biol. 2016;26:1339-45.

27. Harumoto T, Anbutsu H, Fukatsu T. Male-killing Spiroplasma induces sexspecific cell death via host apoptotic pathway. PLoS Pathog. 2014;10:1-10.

28. Paredes JC, Herren JK, Schüpfer F, Lemaitre B. The role of lipid competition for endosymbiont-mediated protection against parasitoid wasps in Drosophila. MBio. 2016;7:1-8.

29. Xie J, Tiner B, Vilchez I, Mateos M. Effect of the Drosophila endosymbiont Spiroplasma on parasitoid wasp development and on the reproductive fitness of wasp-attacked fly survivors. Evol Ecol. 2011:53:1065-79.

30. Ballinger MJ, Perlman SJ. Generality of toxins in defensive symbiosis: ribosome-inactivating proteins and defense against parasitic wasps in Drosophila. PLoS Pathog. 2017;13:1-19.

31. Hamilton PT, Peng F, Boulanger MJ, Perlman SJ. A ribosome-inactivating protein in a Drosophila defensive symbiont. Proc Natl Acad Sci U S A. 2016; 113:350-5.

32. Jaenike J, Unckless R, Cockburn SN, Boelio LM, Perlman SJ. Adaptation via Symbiosis: recent spread of a Drosophila defensive symbiont. Science. 2010; 329:212-5.

33. Endo Y, Tsurugi K. Mechanism of action of ricin and related toxic lectins on eukaryotic ribosomes. Nucleic Acids Symp Ser. 1986;262:187-90.

34. Virgilio M, Lombardi A, Caliandro R, Fabbrini Maria S. Ribosome-inactivating proteins: from plant defense to tumor attack. Toxins. 2010;2:2700-37.

35. Lainhart W, Stolfa G, Koudelka GB. Shiga toxin as a bacterial defense against a eukaryotic predator, Tetrahymena thermophila. J Bacteriol. 2009;191:5116-22.

36. Bergan J, Dyve L, Anne B, Simm R, Skotland T, Sandvig K. Shiga toxins. Toxicon. 2012;60:1085-107

37. Szewczak AA, Moore PB. The Sarcin/ricin loop, a modular RNA. J Mol Biol. 1995;247:81-98.

38. Paredes JC, Herren JK, Schüpfer F, Marin R, Claverol S, Kuo C-H, et al. Genome sequence of the Drosophila melanogaster male-killing Spiroplasma strain MSRO endosymbiont. MBio. 2015:6:1-12.

39. Le Page DP, Metcalf JA, Bordenstein SR, On J, Perlmutter JI, Shropshire JD, et al. Prophage WO genes recapitulate and enhance Wolbachia-induced cytoplasmic incompatibility. Nature. 2017;543:243-7.

40. Duffy JB. GAL4 system indrosophila: a fly geneticist's swiss army knife. Genesis. 2002:34:1-15.

41. Soejima $Y$, Lee, Jae $M$, Nagata $Y$, Mon $H$, liyama $K$, Kitano $H$, et al. Comparison of signal peptides for efficient protein secretion in the baculovirus-silkworm system. Cent Eur J Biol. 2013;8:1-7.

42. Robert BK, Subinay G, Monica A, Sandra G, Allan S, Carol S, et al. Heavy chain dimers as well as complete antibodies are efficiently formed and secreted from Drosophila via a BiP-mediated pathway. J Biol Chem. 1998; 273:20533-8.

43. Iyer J, Wang Q, Le T, Pizzo L, Grönke S, Ambegaokar SS, et al. Quantitative assessment of eye phenotypes for functional genetic studies using Drosophila melanogaster. G3: genes|genomes|. Genetics. 2016:6:1427-37.

44. Halder G, Callaerts P, Flister S, Walldorf U, Kloter U, Gehring WJ. Eyeless initiates the expression of both sine oculis and eyes absent during Drosophila compound eye development. Development. 1998;125:2181-91. 
45. Van-Vactor DL, Cagan RL, Krämer H, Zipursky SL. Induction in the developing compound eye of Drosophila: multiple mechanisms restrict $R 7$ induction to a single retinal precursor cell. Cell. 1991;67:1145-55.

46. Ramond $\mathrm{E}$, Meister M, Lemaitre B. From embryo to adult: hematopoiesis along the Drosophila life cycle. Dev Cell. 2015;33:367-8.

47. Horn L, Leips J, Starz-Gaiano M. Phagocytic ability declines with age in adult Drosophila hemocytes. Aging Cell. 2014;13:719-28.

48. Ashburner M. Drosophila: a laboratory handbook and manual; 1989

49. Bretscher AJ, Honti V, Binggeli O, Burri O, Poidevin M, Kurucz É, et al. The nimrod transmembrane receptor eater is required for hemocyte attachment to the sessile compartment in Drosophila melanogaster. Biology Open. 2015;4:355-63.

50. Livak KJ, Schmittgen TD. Analysis of relative gene expression data using real-time quantitative $P C R$ and the $2-\Delta \Delta C T$ method. Methods. 2001;25: 402-8.

Ready to submit your research? Choose BMC and benefit from:

- fast, convenient online submission

- thorough peer review by experienced researchers in your field

- rapid publication on acceptance

- support for research data, including large and complex data types

- gold Open Access which fosters wider collaboration and increased citations

- maximum visibility for your research: over $100 \mathrm{M}$ website views per year

At BMC, research is always in progress.

Learn more biomedcentral.com/submissions 